\title{
Personalized therapies in psychiatry: promises, pitfalls and perspectives
}

\author{
Katharina Domschke • Daniel J. Müller • \\ Alessandro Serretti
}

Published online: 5 December 2014

(C) Springer-Verlag Wien 2014

\begin{abstract}
What physicians say of distinct species [of melancholy] in their books it much matters not, since that in their patients' bodies they are commonly mixed. In such obscurity, therefore, variety and confused mixture of symptoms, causes, how difficult a thing is it to treat of several kinds apart; to make any certainty or distinction among so many casualties, distractions, when seldom two men shall be like effected per omnia! 'Tis hard, I confess, yet nevertheless I will adventure through the midst of these perplexities, and, led by the clue or thread of the best writers, extricate myself out of a labyrinth of doubts and errors, and so proceed to the causes.
\end{abstract}

Robert Burton, "The Anatomy of Melancholy", 1651, Sect. I, Memb. 3, Subsect. 4

What has very astutely been said about the highly heterogeneous etiology and symptomatology of "melancholy"

K. Domschke $(\bowtie)$

Department of Psychiatry, Psychosomatics and Psychotherapy, Center of Mental Health, University of Wuerzburg, Wuerzburg, Germany

e-mail: Domschke_K@ukw.de

D. J. Müller

Campbell Family Mental Health Institute, Centre for Addiction and Mental Health, Toronto, Canada

D. J. Müller

Department of Psychiatry, Institute of Medical Science, University of Toronto, Toronto, Canada

A. Serretti

Department of Biomedical and NeuroMotor Sciences,

University of Bologna, Bologna, Italy by Robert Burton as early as in 1651 is still true today and is not only true for major depression but for all complexgenetic neuropsychiatric disorders. Since "seldom two men shall be like effected per omnia", disentangling the "mixture" of etiological factors and clinical presentations on an individual level is essential for understanding the unique phenomenology of the single patient's disorder and-based on this knowledge-for risk assessment and prediction, for targeted prevention, for early detection and diagnosis as well as for "treat[ing] of several kinds apart", i.e., for personalized and thus more effective and safer treatment approaches individually tailored to the individual patient's constellation of risk factors.

In the present special issue on "Personalized Therapies in Psychiatry: State of the Art and Perspectives", we are going to "adventure through the midst of these perplexities" having accumulated "clues or threads" on multiple facets of personalized medicine in psychiatry.

A wide range of methodologies applied to test potential (bio)markers of major neuropsychiatric disorders and their treatment is presented beginning with classical pharmacogenetic approaches targeting pharmacokinetic, i.e., CYP 450 gene systems (Brandl et al. this issue; Gressier et al. this issue; for review see Spina and de Leon, this issue) in psychosis and depression. Although CYP pharmacogenetic testing has already become available in some clinical settings (cf. Müller et al. 2013), such services are not established yet as common practice. Therefore, the present papers may further inform on the potential value and clinical applicability of CYP gene testing.

Furthermore, research into biomarker-based prediction of therapy response extends to tolerability and side effects. Accordingly, exploiting large datasets Fabbri et al. (this issue) conducted a genetic study on the side effects of psychotropic medications in patients with bipolar disorder. 
Environment is another important variable to be considered in neuropsychiatric disorders and their treatment. In this issue, Chang et al. illustrate gene-environment interaction, i.e., an interaction of proopiomelanocortin gene variation and stressful life events, in mediating antidepressant treatment response.

Beyond the traditional investigation of few common single nucleotide polymorphisms (SNP) in pharmacogenetic studies, deeper methods of analysis are needed. Recent methodological and conceptual developments in the field of genetics such as next generation sequencing (see Goldman and Domschke 2014) and epigenetics/pharmacoepigenetics (see Menke and Binder 2014) are reflected by a review article on genomic structural variation including copy number variation (CNV) in affective, anxiety and stress-related disorders (Ono et al. this issue), a review article on microRNAs in mediating risk and therapy response in neuropsychiatric disorders (Hommers et al. this issue) and first results on DNA methylation as a possible epigenetic predictor of therapy response in major depression (Domschke et al. this issue).

Beyond genetic/epigenetic mechanisms, treatment effects largely depend on subtle variations on a systems level. In an in vitro cell model probing the impact of lithium in combination with different antidepressants on the immune system, Petersein et al. (this issue) report lithium to increase pro-inflammatory cytokine levels and discuss the potential of immunological markers to guide individualized treatment decisions in affective disorders.

Neuroimaging, particularly when combined with genetic data employing an imaging genetics approach (for review see Domschke and Dannlowski 2010), offers new venues in enhancing the power of research into biomarkers and individualized treatment, as it allows for an unprecedented resolution in investigating the pathophysiology of disease and treatment. In the present issue, two papers by Lueken et al. exemplarily present imaging and combined imaging genetic markers emerging from structural and functional MRI studies in anxiety disorders, which may aid in informing nosological classifications as well as psychotherapeutic treatment decisions.

Finally, the rapid growth and increasing intricacy of molecular and imaging techniques must be escorted by the development of correspondingly innovative and complex bioinformatic approaches. In this issue, Cocchi et al. exemplarily propose an algorithm for interactive, i.e., epistatic effects of several risk variants emerging from genome-wide association studies (GWAS) combined with clinical covariants. Along similar lines and concluding this issue, Clark et al. present a mathematical model providing a tool to integrate clinical, neurocognitive, neuroimaging and electrophysiological data in the attempt to develop combined markers for indicated prevention in psychosis.
Despite these promising studies illustrating an increasing wealth of data on biomarkers of personalized diagnostics and treatment of neuropsychiatric disorders, the field is still troubled with a "labyrinth of doubts and errors". Substantial challenges remain such as not invariably replicable data, gender and ethnicity as additional covariates, phenomenological subtypes and "big data" as for instance emerging from genome-wide association studies. Regarding complex-genetic neuropsychiatric disorders and their treatment, research into personalized medicine would want to identify a panel of multidimensional biomarkers emerging from different technologies at the intersection of pharmacology, imaging and genetics combined with clinical data, which entails intensive crossdisciplinary collaborations between psychiatrists, psychologists, biologists/geneticists, physicists and pharmacologists (cf. Gerretsen et al. 2009; Kennedy et al. 2012). Correspondingly, integrative biostatistical approaches modeling the intricate interplay of a multitude of biological, environmental and clinical factors are urgently needed. Also, cost-utility analyses of pharmacogenetic/biomarker testing are warranted to evaluate the individual as well as global socioeconomic benefit of a personalized treatment approach as exemplarily probed by the C.E.A.P. (CostEffectiveness Analysis on Psychiatric Disorders) group (cf. Olgiati et al. 2013). Consensus guidelines will be necessary to inform clinicians on which biomarkers are useful and how they can be implemented in daily clinical practice (cf. Drozda et al. 2014). At the patient level, education and teaching regarding advances and limitations in the field are germane to a realistic understanding of the benefits of personalized medicine. One particular aspect here will be to inform patients that, despite receiving the same treatment, outcome may change over time in a given individual and that personalized medicine is not an "entity of reliability, but an entity of probability" (Kalow 2007).

In summary, the promise of all endeavors in the field of personalized medicine research is a more person-centered and thus eventually more humanistic diagnostic and therapeutic approach. While this term is classically tied to a holistic view of the patient emphasizing human uniqueness and individual potential as opposed to the more schematic classification of research approaches, it may gain an extended meaning by integrating multiple biological and clinical markers in a reproducible, evidence-based way. This is hoped to aid in developing early and targeted preventive interventions and to reduce the individual patient's suffering by means of more effective and tolerable therapeutic interventions, which at the same time will lower the socioeconomic burden of neuropsychiatric disorders. It should be noted that these advances in identifying individual risk factors and markers of treatment response have to be accompanied by strict national and international 
regulations about stigmatization, privacy confidentiality and data protection (cf. Serretti and Artioli 2006).

\section{References}

Burton R (1651) The anatomy of melancholy. The New York Review of Books, New York

Domschke K, Dannlowski U (2010) Imaging genetics of anxiety disorders. NeuroImage 53:822-831

Drozda K, Müller DJ, Bishop JR (2014) Pharmacogenomic testing for neuropsychiatric drugs: current status of drug labeling, guidelines for using genetic information, and test options. Pharmacotherapy 34:166-184

Gerretsen P, Müller DJ, Tiwari A, Mamo D, Pollock BG (2009) The intersection of pharmacology, imaging, and genetics in the development of personalized medicine. Dialogues Clin Neurosci 11:363-376

Goldman D, Domschke K (2014) Making sense of deep sequencing. Int J Neuropsychopharmacol 17:1717-1725
Kalow W (2007) Personalized medicine-some thoughts. McGill J Med 10:58

Kennedy SH, Downar J, Evans KR, Feilotter H, Lam RW, MacQueen GM, Milev R, Parikh SV, Rotzinger S, Soares C (2012) The Canadian Biomarker Integration Network in Depression (CANBIND): advances in response prediction. Curr Pharm Des 18:5976-5989

Menke A, Binder EB (2014) Epigenetic alterations in depression and antidepressant treatment. Dialogues Clin Neurosci 16:395-404

Müller DJ, Kekin I, Kao AC, Brandl EJ (2013) Towards the implementation of CYP2D6 and CYP2C19 genotypes in clinical practice: update and report from a pharmacogenetic service clinic. Int Rev Psychiatry 25:554-571

Olgiati P, Bajo E, Bigelli M, Montgomery S, Serretti A, C.E.A.P (Cost-Effectiveness Analysis on Psychiatric Disorders)-group (2013) Challenging sequential approach to treatment resistant depression: cost-utility analysis based on the Sequenced Treatment Alternatives to Relieve Depression (STAR*D) trial. Eur Neuropsychopharmacol 23:1739-1746

Serretti A, Artioli P (2006) Ethical problems in pharmacogenetic studies of psychiatric disorders. Pharmacogenomics J6:289-295 